\title{
Favorable outcome of plasmablastic lymphoma expressing both immunoglobulin light chains arising in an immunocompetent man
}

\author{
Hien K. Duong • Claudiu V. Cotta
}

Received: 20 March 2012 / Accepted: 4 May 2012 / Published online: 27 May 2012

(C) Springer-Verlag 2012

\begin{abstract}
This case illustrates the diagnostic and therapeutic challenges posed by neoplasms with plasmablastic features. Even though differentiating between plasmablastic lymphoma (PL) and plasma cell neoplasms can be difficult and somewhat arbitrary, the implications for the therapeutic regimen are significant. In this case, the lesion was identified in the nasal cavity of a previously healthy 37 -year-old man, HIV negative. While the neoplasm had all the features of PL, including positivity for Epstein-Barr virus, it also showed sheets of monotypic kappa cells alternating with sheets of monotypic lambda cells. Molecular studies showed a population with clonally rearranged IGH and peaks corresponding to IGK VJ and IGK VKdel. The patient was treated with 3 cycles of rituximab, cyclophosphamide, vincristine, doxorubicin, and prednisone, with excellent results. The patient is free of disease more than 20 months later. In the absence of randomized trials investigating the effectiveness of therapeutic protocols for PL, currently aggressive regimens are recommended. This case shows that at
\end{abstract}

This study has been performed in accordance with the provisions of the Institutional Review Board.

H. K. Duong

Department of Hematologic Oncology and Blood Disorders,

Taussig Cancer Institute, Cleveland Clinic,

Cleveland, $\mathrm{OH}, \mathrm{USA}$

C. V. Cotta

Pathology and Laboratory Medicine Institute, Cleveland Clinic, Cleveland, OH 44195, USA

C. V. Cotta $(\triangle)$

Laboratory Medicine/L-11, Cleveland Clinic,

9500 Euclid Avenue,

Cleveland, OH 44195, USA

e-mail: cottac@ccf.org least in a subset of the patients with PL, a less aggressive approach may be considered. It is also the first case of PL expressing both light chains.

Keywords Highly active antiretroviral therapy · Plasmablastic lymphoma · Immunoglobulin light chains · Plasma cell neoplasms · Epstein-Barr virus . Hematolymphoid neoplasms

\section{Introduction}

According to the WHO 2008 classification of hematolymphoid neoplasms, plasmablastic lymphomas (PL) are diffuse proliferations of large neoplastic cells, morphologically resembling immunoblasts, but with an immunophenotype corresponding to plasma cells. Lesions with these characteristics were initially observed in the oral cavities of severely immunosuppressed patients, usually HIV positive. These patients had no other evidence of plasma cell neoplasms ( $\mathrm{PCN})$, and the lesions did not respond to therapeutic regimens directed either to lymphoma or to PCN. This characteristic presentation, aggressive clinical course, and poor outcome in spite of the therapeutic regimens were thought to be indicative of tumor pathobiology different than that of PCN, more close to that of lymphomas, leading to the creation of a new diagnostic entity. However, from the beginning differentiating between PL and PCN was difficult, relying heavily on the clinical history, and in a few cases, it was rather arbitrary. Reports of PL arising in immunocompetent patients further complicated the issue, decreasing the value of the clinical presentation as a diagnostic tool. The observation that most cases diagnosed as PL were positive for Epstein-Barr virus (EBV) somewhat simplified the task 
of the pathologist, but how reliable this finding is as a diagnostic criterion it still a matter of debate. In the therapeutic field, the only significant progress is the introduction of highly active antiretroviral therapy (HAART). This was shown to improve the prognosis of many cases of PL when immunosuppression was secondary to HIV. However, as most cases of PL are still thought to have a poor prognosis and in the absence of rigorous studies, most patients are treated with aggressive regimens.

\section{Clinical history}

The patient is a 35-year-old male who presented with rhinorrhea and difficulty breathing through his nostrils. He had no prior medical history and was not on any medications. He reported no fatigue, weight loss, fevers, or night sweats. The physical examination was unremarkable; he did not have any lymphadenopathy or organomegaly. He was initially treated with antibiotics and nasal steroids; however, the symptoms persisted. Computed tomography of the sinuses revealed mild mucosal thickening of the right frontal sinus. The patient subsequently underwent endoscopic examination and was found to have a small, partially obstructing mass, extending from the inferior turbinate within the right nasal cavity.

\section{Materials and methods}

\section{Immunohistochemistry}

Stains were performed on $4-\mu m$-thick sections from the formalin-fixed, paraffin-embedded tissue block. The antibodies used were CD20 [Dako, Carpinteria, CA, USA (DC), Cat. no. M0755], CD30 (DC, Cat. no. M0751), CD56 (Novocastra, Newcastle Upon Tyne, UK, Cat. no. CD56-1B6), CD79a (DC, Cat. no. 7050), CD138 (Biocare, Concord, CA, USA, Cat. no. CM197C), Ki-67 [Ventana Medical Systems, Tucson, AZ, USA (VMS), Cat. no. 7904286], P53 (DC, Cat. no. M7001), and Pax5 (BD Transduction, San Jose, CA, USA, Cat. no. 610863). Automated immunostainers (Benchmark XT and Ultra, VMS) were used. For detections, the iView and UltraView DAB Detection (VMS) kits were used.

Chromogenic in situ hybridization

Sections from the fixed, paraffin-embedded tissue were stained using a Benchmark XT (VMS) automatic stainer and the VMS reagents for EBV-encoded small RNA (EBER; Cat. no. 760-1209), kappa (Cat. no. 780-2843), or lambda (Cat. no. 780-2844).
Fluorescence in situ hybridization

After specimen probe processing, the LSI MYC dual-color break-apart probe was used (Abbott Molecular, Des Plains, IL, USA, Cat. No 05J91-001).

\section{Molecular studies}

After DNA extraction, polymerase chain reaction was performed following the guidelines of the BIOMED-2 consortium, with fluorescently labeled primers (Invivoscribe, San Diego, CA, USA). The products of amplification were detected with an ABI 3730 genetic analyzer (Applied Biosystems, Carlsbad, CA, USA).

Results of pathological examination and treatment

An excisional biopsy was performed, and histologic examination revealed sheets of large cells with plasmacytoid features and underlying unremarkable respiratory mucosa (Fig. 1). These cells had abundant cytoplasm and round or oval nuclei, with central conspicuous nucleoli. In a few cells, the nuclei had invaginations with cytoplasmic content- "Dutcher bodies" (Fig. 1). The mitotic activity of the neoplastic cells was intermediate to high, there were three to four mitotic figures/high-power field. Immunohistochemical stains showed these cells to have an immunophenotype corresponding to plasma cells, positive for CD138 and CD56 (Fig. 2c, d), but negative for CD20, CD30, and Pax5 (Fig. 2a, b). Chromogenic in situ hybridization (CISH) with probes for EBER showed most cells to be positive (Fig. 2g), while the stain for human herpes virus 8 was negative. An unusual result was noticed with CISH with probes for mRNA encoding for kappa or lambda

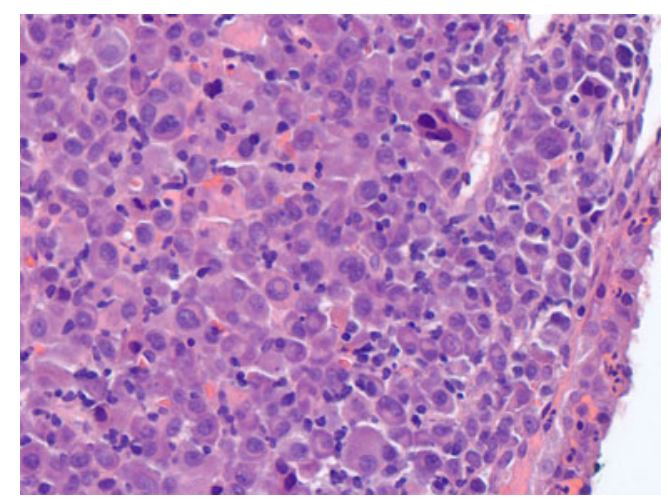

Fig. 1 Histologic section of the lesion, stained with hematoxylin and eosin. Original magnification, $\times 400$. Sheets of large cells, with abundant cytoplasm and single or multiple round nuclei, with central eosinophilic nucleoli. One Dutcher body is visible in the center of the image. Respiratory epithelium is represented in the lower right corner of the specimen 
Fig. 2 Special stains. a Immunohistochemical stain for CD20, original magnification, $\times 200$. The neoplastic cells are negative. b Pax5, $\times 200$. The stain is negative. $\mathbf{c}$ The antibody for $\mathrm{CD} 138$ shows the cells and the epithelium to be positive, $\times 200$. d Expression of CD56 by the neoplastic cells, $\times 200$. e The mitotic activity as shown by the antibody for Ki-67 is relatively high, $\times 200$. f The neoplastic cells show dim positivity for p53, $\times 200$. g EBER, $\times 200$. h CISH for kappa. The fragment on the right is composed of sheets of positive cells, $\times 20$. i CISH for lambda. The fragment on the left is composed of sheets of positive cells, $\times 20$

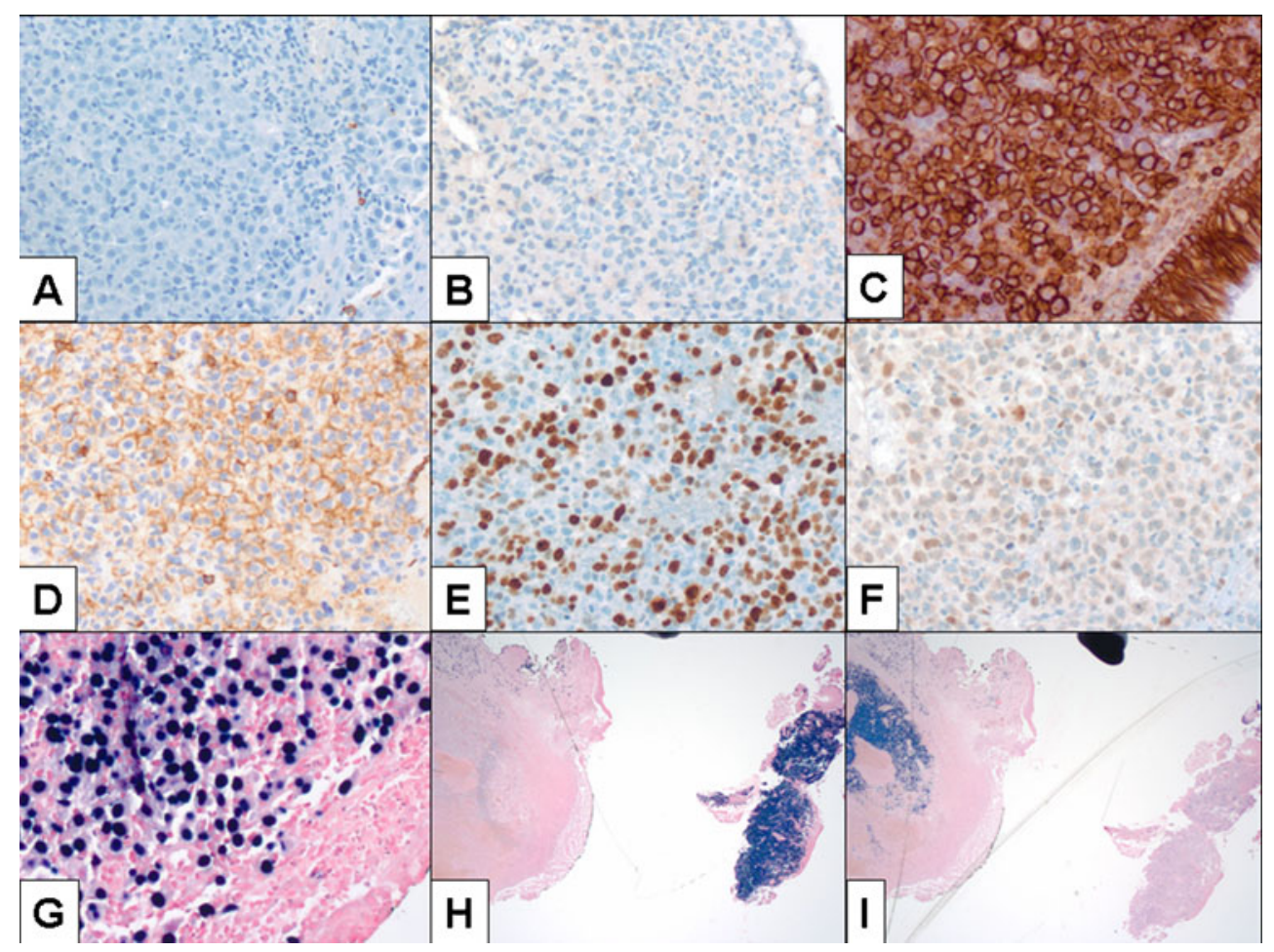

immunoglobulin light chains: sheets of cells positive for kappa alternated with areas in which cells positive for lambda predominated, indicating a biclonal lesion (Fig. 2h, i).

Additional molecular studies were performed. The polymerase chain reaction performed on the DNA extracted from the fixed, paraffin-embedded block with primers for the immunoglobulin genes (BIOMED-2) showed at least one population of cells with clonally rearranged immunoglobulin genes. All three primer combinations amplifying IGH VDJ (FR1-FR3) showed only one dominant amplicon. Interestingly, similar results were shown by the primers for IGK VJ and for IGK VKde, indicating one clonal population, the possible presence of two cell populations sharing the same heavy chain rearrangement but one with kappa and the other one with lambda light chain rearrangements, or of a population with biallelic rearrangements. Fluorescence in situ hybridization studies were negative for translocations involving IgH (14q32) or MYC (8q24), but over $40 \%$ of the malignant cells showed gain of genetic material at $8 \mathrm{q} 24$ or aneusomy 8.

After the diagnosis of EBV-associated plasmablastic neoplasm was made, the differential diagnosis included plasmablastic lymphoma, extranodal marginal zone lymphoma of mucosa-associated lymphoid tissue (MALT lymphoma) with prominent plasmacytic differentiation, plasma cell myeloma, and extraosseous plasmacytoma. Tests for human immunodeficiency virus were negative, and there was no clinical or immunologic evidence of an immunodeficiency, which did not help further clarify the diagnosis. The complete absence of cells positive for CD20 or for Pax 5 was considered inconsistent with MALT lymphoma. Extensive imaging studies conducted by computed tomography, magnetic resonance imaging, or positron emission tomography scans showed no residual tumor, adenopathy, lytic bone lesions, or other abnormality. The bone marrow biopsy was negative for a malignant process, while repeated serum and urine electrophoresis studies showed no evidence of a monoclonal protein, which ruled out the possibility of plasma cell myeloma. Differentiation between extraosseous plasmacytoma with plasmablastic features from plasmablastic lymphoma is very difficult, and there are no reliable criteria that differentiate between the two lesions in an immunocompetent patient. However, given the positivity for EBV, in this case the diagnosis of plasmablastic lymphoma was favored.

Following the diagnosis, the patient completed 3 cycles of combined chemotherapy with rituximab, cyclophosphamide, vincristine, doxorubicin, and prednisone, which was well tolerated. Given the location of his mass and the risk of central nervous system involvement, he also received four prophylactic doses of intrathecal chemotherapy. He then underwent involved-field radiotherapy. Twenty months after the completion of his treatment, the patient continues to show no evidence of residual disease.

\section{Discussion}

Plasmablastic neoplasms are rare entities that give rise to multiple diagnostic and therapeutic challenges. The term 
plasmablastic was initially used by hematologists to identify a subset of plasma cell myelomas with a characteristic blastic morphology and an aggressive clinical course [1-3]. Later on, the term plasmablastic lymphoma was also used to describe lesions arising in immunosuppressed patients, usually HIV-positive patients, involving areas surrounding the oral cavity [4]. These lesions represented approximately $2.6 \%$ of lymphomas associated with acquired immunodeficiency syndrome and were associated with positivity of the neoplastic cells for EBV [5, 6]. More recently, several studies have been published in HIV-negative patients, although still in immunocompromised conditions such as post-solid organ or bone marrow transplant, autoimmune disorders, or other malignancies [7-9].

Histopathologically, plasmablastic lesions are composed of sheets of large cells, with moderate to abundant cytoplasm and round or oval nuclei, with conspicuous central nucleoli [10]. The immunophenotype of these cells is similar to that of plasma cells, most B-lineage markers not being detected [10]. Whether these lesions were classified as lymphomas or plasma cell neoplasms, regardless of the therapy received, most cases had a poor prognosis [4, 11-13]. The combination of immunosuppression, typical perioral presentation, aggressive clinical behavior, and failure to respond to therapies designed for plasma cell myeloma suggested that in spite of the significant phenotypic overlap with other plasma cell neoplasms, these plasmablastic lesions should be considered a separate entity with biologic characteristics closer to an aggressive lymphoma than to myeloma.

Gene expression profile studies have shown contradicting results as some studies show plasmablastic lymphomas to have a gene expression profile similar to that of diffuse large B cell lymphomas, while others point to similarities with plasma cell myeloma $[14,15]$. In most cases, genetic abnormalities involving MYC have been demonstrated, mainly by fluorescence in situ hybridization [16-18]. Recently, positivity for EBV emerged as a common feature of cases diagnosed as plasmablastic lymphoma, in contrast with the cases diagnosed as plasmablastic myeloma, which were EBV negative [19].

As expected, clinicians treating patients with plasmablastic neoplasms have to decide whether to use regimens for plasma cell neoplasms or for lymphoma. The literature on this topic is scant, and there are no prospective randomized trials to investigate the effectiveness of different regimens. At least $50 \%$ of the cases in the literature have been treated with CHOP or related regimens [20, 21]. Currently, the National Comprehensive Cancer Network guidelines recommend treatment with more intensive regimens, such as EPOCH, HyperCVAD, or CODOX-M/IVAC [20]. Autologous stem cell transplant has also been implemented in upfront therapy [9]. These intensive regimens have been advocated mainly due to poor long-term survival and aggressive behavior of the disease suggested by the abnormalities involving MYC or the non-germinal center immunophenotypic profile of the neoplastic cells. The limited available data show no definitive survival advantage of these more aggressive regimens [21]. Bortezomib has also been explored in a small number of cases, but to date, there is no conclusion regarding its usefulness [22, 23].

As more effective therapies for patients with HIV have been developed, the prognosis of these patients became influenced by the management of their infectious complications $[7,21]$. In fact, in a small number of cases, HAART led to complete remission even in the absence of additional chemotherapy $[9,24]$. Interestingly in a small study, positivity for HIV followed by treatment for infection was associated with a better prognosis than negativity for HIV, pointing to the possible role of immunodeficiencies of other etiologies [7].

The prognosis of patients with plasmablastic lymphoma is thought to be poor, even if over $75 \%$ of these patients respond to chemotherapy [7]. In the absence of treatment, the median survival of patients is approximately 3 months [7]. When treated, $46 \%$ of patients achieve complete remission, while $31 \%$ show only a partial response. Patients with complete response show a longer survival (11 months) than those with partial response (3.5 months). Identification of translocations involving MYC, of IGH/MYC in particular, seems to be associated with a poorer prognosis, with an overall survival of 3 months [15]. In general, older patients ( $>60$ years) and advanced stage, including bone marrow involvement, are thought to be indicators of poor prognosis $[7,21]$.

We present an unusual case of plasmablastic lymphoma arising in a young immunocompetent male. At presentation, the lesion was located in the nasal pharyngeal cavity, and the patient had no constitutional symptoms. Histologic and immunophenotypic analysis showed all the features described in plasmablastic lymphomas: positivity for plasma cell markers and EBV and negativity for B-lineage markers. Morphologically, some of the neoplastic cells were closer to plasma cells than to plasmablasts, a feature that has been previously described [12]. The lesion was composed of separate sheets of monotypic kappa and monotypic lambda cells; an unusual feature in hematolymphoid neoplasms, to our knowledge never before reported in PL. The patient underwent combined modality treatment with chemotherapy and involved field radiation therapy, remaining disease-free.

Literature search shows that this is the first case of plasmablastic lymphoma expressing both light chains ever reported. Other unusual features of this case include immunocompetent status of the patient, the location in the nasal cavity, and the excellent sustained response to therapy. While the conclusions from this case cannot be generalized in the absence of reliable clinical studies, they indicate that a 
cautious, conservative approach should at least be considered in the management of neoplasms with plasmablastic morphology that present in immunocompetent patients.

Conflict of interest The authors have no conflict of interest to declare.

\section{References}

1. Bartl R, Frisch B, Burkhardt R et al (1982) Bone marrow histology in myeloma: its importance in diagnosis, prognosis, classification and staging. Br J Haematol 51:361-375

2. Greipp PR, Leong T, Bennett JM et al (1998) Plasmablastic morphology - an independent prognostic factor with clinical and laboratory correlates: Eastern Cooperative Oncology Group (ECOG) myeloma trial E9486 report by the ECOG Myeloma Laboratory Group. Blood 91:2501-2507

3. Greipp PR, Raymond NM, Kyle RA, O'Fallon WM (1985) Multiple myeloma: significance of plasmablastic subtype in morphological classification. Blood 65:305-310

4. Delecluse HJ, Anagnostopoulos I, Dallenbach F et al (1997) Plasmablastic lymphomas of the oral cavity: a new entity associated with the human immunodeficiency virus infection. Blood 89:1413-1420

5. Carbone A, Gaidano G, Gloghini A, Ferlito A, Rinaldo A, Stein H (1999) AIDS-related plasmablastic lymphomas of the oral cavity and jaws: a diagnostic dilemma. Ann Otol Rhinol Laryngol 108:95-99

6. Carbone A, Gloghini A (2008) Plasmablastic lymphoma: one or more entities? Am J Hematol 83:763-764

7. Castillo JJ, Winer ES, Stachurski D et al (2010) Clinical and pathological differences between human immunodeficiency virus-positive and human immunodeficiency virus-negative patients with plasmablastic lymphoma. Leuk Lymphoma 51:2047-2053

8. Kim JE, Kim YA, Kim WY et al (2009) Human immunodeficiency virus-negative plasmablastic lymphoma in Korea. Leuk Lymphoma 50:582-587

9. Liu JJ, Zhang L, Ayala E et al (2011) Human immunodeficiency virus (HIV)-negative plasmablastic lymphoma: a single institutional experience and literature review. Leuk Res 35:1571-1577

10. Swerdlow SHCE, Harris NL, Jaffe ES, Pileri SA, Stein H, Thiele J, Vardiman JW (eds) (2008) WHO classification of tumours of haematopoietic and lymphoid tissues. IARC, Lyon
11. Castillo J, Pantanowitz L, Dezube BJ (2008) HIV-associated plasmablastic lymphoma: lessons learned from 112 published cases. Am J Hematol 83:804-809

12. Colomo L, Loong F, Rives S et al (2004) Diffuse large B-cell lymphomas with plasmablastic differentiation represent a heterogeneous group of disease entities. Am J Surg Pathol 28:736-747

13. Dong HY, Scadden DT, de Leval L, Tang Z, Isaacson PG, Harris NL (2005) Plasmablastic lymphoma in HIV-positive patients: an aggressive Epstein-Barr virus-associated extramedullary plasmacytic neoplasm. Am J Surg Pathol 29:1633-1641

14. Chang CC, Zhou X, Taylor JJ et al (2009) Genomic profiling of plasmablastic lymphoma using array comparative genomic hybridization $(\mathrm{aCGH})$ : revealing significant overlapping genomic lesions with diffuse large B-cell lymphoma. J Hematol Oncol 2:47

15. Taddesse-Heath L, Meloni-Ehrig A, Scheerle J, Kelly JC, Jaffe ES (2010) Plasmablastic lymphoma with MYC translocation: evidence for a common pathway in the generation of plasmablastic features. Mod Pathol 23:991-999

16. Bogusz AM, Seegmiller AC, Garcia R, Shang P, Ashfaq R, Chen W (2009) Plasmablastic lymphomas with MYC/IgH rearrangement: report of three cases and review of the literature. Am J Clin Pathol 132:597-605

17. Dawson MA, Schwarer AP, McLean C et al (2007) AIDS-related plasmablastic lymphoma of the oral cavity associated with an IGH/ MYC translocation - treatment with autologous stem-cell transplantation in a patient with severe haemophilia-A. Haematologica 92:e11-12

18. Valera A, Balague O, Colomo L et al (2010) IG/MYC rearrangements are the main cytogenetic alteration in plasmablastic lymphomas. Am J Surg Pathol 34:1686-1694

19. Vega F, Chang CC, Medeiros LJ et al (2005) Plasmablastic lymphomas and plasmablastic plasma cell myelomas have nearly identical immunophenotypic profiles. Mod Pathol 18:806-815

20. 3-2011. NCCNNPGiOA-rB-clV. 2012

21. Castillo JJ, Winer ES, Stachurski D et al (2010) Prognostic factors in chemotherapy-treated patients with HIV-associated Plasmablastic lymphoma. Oncologist 15:293-299

22. Bibas M, Grisetti S, Alba L, Picchi G, Del Nonno F, Antinori A (2010) Patient with HIV-associated plasmablastic lymphoma responding to bortezomib alone and in combination with dexamethasone, gemcitabine, oxaliplatin, cytarabine, and pegfilgrastim chemotherapy and lenalidomide alone. J Clin Oncol 28:e704-708

23. Bose P, Thompson C, Gandhi D, Ghabach B, Ozer H (2009) AIDS-related plasmablastic lymphoma with dramatic, early response to bortezomib. Eur J Haematol 82:490-492

24. Armstrong R, Bradrick J, Liu YC (2007) Spontaneous regression of an HIV-associated plasmablastic lymphoma in the oral cavity: a case report. J Oral Maxillofac Surg 65:1361-1364 